\title{
A INTEGRAL INICIAL
}

Eleni Bisognin

Departamento de Matemática. Centro de Ciēncias Naturais e Exatas. UFSM. Santa Maria, RS.

RESUMO

Neste trabalho define-se o que se entende por integral in $i$ cial e demonstra-se que a integral de Riemann sobre o conjunto $R([a, b]$; $\mathbb{R}$ ) das funçōes definidas no intervalo $[a, b]$, integrāveis, $\bar{e}$ uma in tegral inicial.

A seguir demonstra-se o teorema da convergência dominada de Arzelà no contexto da teoria de Riemann.

SUMMARY

BISOGNIN,E., 1981. The initial integral. Ciência e Natura (3):5-11.

The pourpouse of this work is to define what is understood by initial integral and also to demonstrate that Riemann's integral over the set $R([a, b] ; \mathbb{R})$ of the integrable defined functions in the compact interval [a,b], is an initial integral.

The Arzelà's Dominated Convergence Theorem in Riemann's theory context is demonstrated.

INTRODUÇÃO

As integrais clāssicas, como por exemplo a integral de Riemann, gozam da propriedade de passagem ao limite. Seguindo as no ções apresentadas por Luxemburg (4) é dada uma versão do teorema da convergência dominada, cujas idēias iniciais foram apresentadas por Arzelà (1) no contexto da teoria de Riemann.

Neste trabalho foi feito o estudo preliminar demonstrando que a integral de Riemann è uma integral inicial.

\section{REVISÄO DA LITERATURA}

Seja $X$ um conjunto qualquer e $F(X, \mathbb{R})$ o conjunto de todas as funções reais definidas em $X$. Dizemos que $E C F(X, \mathbb{R}) \bar{e}$ um clã se as seguintes condições são verificadas:

a) E é um subespaço vetorial de $F(X ; \mathbb{R})$.

b) Toda função f€E é $T$ imitada.

c) Se $F \in E$ e $g: \mathbb{R} \rightarrow \mathbb{R}$ é uma função contínua com $g(0)=0$, então gof $\in E$. Como exemplo de clãs temos:

a) 0 conjunto. $C([a, b] ; \mathbb{R})$ das funções reais continuas no intervalo $[a, b]$.

b) 0 conjunto $E s c([a, b] ; \mathbb{R})$ das funções reais escalonadas no inter va $10[a, b]$. 
c) 0 conjunto $\operatorname{Reg}([a, b] ; \mathbb{R})$ das funções reais reguladas, definidas em $[a, b]$. (Dizemos que uma função $f$ definida no intervalo $[a, b] \bar{e}$ uma função regulada se existe o limite à direita $f(x+)$ para todo $x \in[a, b[$ e existe o limite à esquerda $f(x-)$ para todo $x \in] a, b]$ ).

d) 0 conjunto $D([a, b] ; \mathbb{R})$ das funções reais limitadas com um nūmero finito de descontinuidades em $[\mathrm{a}, \mathrm{b}]$.

$$
\text { A verificação de que os conjuntos a) b) c) d) constituem }
$$

clãs ē simples. Estes conjuntos são também clãs de funções integrä veis à Riemann.

e) 0 conjunto $R([a, b] ; \mathbb{R})$ das funções reais 1 imitadas, definidas em $[a, b]$, integrāveis à Riemann, é um clã de funções que contém os clãs anteriores.

A demonstração de que $R([a, b] ; \mathbb{R})$ é um clã encontra-se em (2) no Teorema 2.4 .

f) 0 conjunto $C(X ; \mathbb{R})$ de todas as funções reais, continuas, defin $\underline{i}$ das no espaço compacto $X$.

g) 0 conjunto $B C(X ; \mathbb{R})$ de todas as funções reais, contínuas e limi tadas sobre o espaço topológico $X$.

\section{DESEN VOLVIMENTO}

Foi citado anteriormente que a integral de Riemann goza da propriedade de passagem ao limite. Exemplifica-se, desenvolvendo o caso particular da restrição da integral de Riemann ao conjunto $c([\mathrm{a}, \mathrm{b}] ; \mathbb{R})$.

Teorema 1

Seja $\left(f_{n}\right)$ uma sequência decrescente de funções reais con tinuas sobre o intervalo compacto $[a, b]$ pontualmente convergente pa ra zero sobre $[a, b]$. Então $\lim _{n \rightarrow \infty} f_{a}^{b} f_{n}(t) d t=0$.

Demonstrąão

0 teorema de Dini (5), afirma que $\left(f_{n}\right)$ converge para zero uniformemente. Então, pelo teorema 2.2 em (2), 1 im $\int_{a}^{b} f_{n}(t) d t=\int_{a}^{b}(t) d t=0$.

No teorema demonstrado está em jogo o $0^{n \rightarrow \infty}$ uncional linear po sitivo $f \longmapsto \int_{a}^{b} f(t) d t$ sobre ocla $c([a, b] ; \mathbb{R})$.

Teorema 2

Seja $X$ um conjunto e $\operatorname{ECF}(X ; \mathbb{R})$ um espaço vetorial. Se $\mu: E \rightarrow \mathbb{R}$ é um funcional linear positivo, são equivalentes as proposições se guintes:

I) $\operatorname{Se}\left(f_{n}\right) C E$ è uma sequéncia crescente e pontualmente convergente para $f_{0} \in E$, então $\mu\left(f_{n}\right)$ converge para $\mu\left(f_{0}\right)$.

I ) Se $\left(g_{n}\right) C E$ é uma sequência decrescente e pontualmente convergen te para $g_{0} \in E$ então $\mu\left(g_{n}\right)$ converge para $\mu\left(g_{0}\right)$ : 
III) Se $\left(g_{n}\right) C E$ è uma sequência decrescente pontualmente convergente para zero, então $\mu\left(g_{n}\right)$ converge para zero.

IV) $\operatorname{Se}\left(h_{n}\right) C E$ é uma sequência de termos positivos com $\sum_{n=1}^{\infty} h_{n}=h_{0}, h_{0} \in E$, então $\sum_{n=1}^{\infty} \mu\left(h_{n}\right)=\mu\left(h_{0}\right)$.

Demonstração

(I) implica (II). Como $\left(g_{n}\right) C E$ é uma sequência decrescente, então $\left(g_{1}-g_{n}\right) C E$ é uma sequência crescente e $\left(g_{1}-g_{n}\right)$ converge para $\left(g_{1}-g_{0}\right)$. Por $(I)$, segue-se que $\mu\left(g_{1}-g_{n}\right)$ converge para $\mu\left(g_{1}-g_{0}\right)$; 10 go $\mu\left(g_{n}\right)$ converge para $\mu\left(g_{0}\right)$.

(II) implica (III). A condição (III) è um caso particular de (II).

(III) implica (IV). Temos que $\left(h_{0}-\sum_{j=1}^{n} h_{j}\right)$ è uma sequên cia de $E$ que decresce para zero. Por hipötese $\mu\left(h_{0}-\sum_{j=1}^{n} h_{j}\right)$ con $\underset{\infty}{\operatorname{verge}}$ para zero, então $\left(\mu\left(h_{0}\right)-\sum_{j=1}^{n} \mu\left(h_{j}\right)\right)$ converge para zero, isto é, $\sum_{n=1} \mu\left(h_{n}\right)=\mu\left(h_{0}\right)$.

(IV) implica (I). Seja $\sum_{n=1}^{\infty} h_{n}=h_{0} \operatorname{com} h_{n} \geq 0$, para todo $n$ tal que $\sum_{n=1}^{\infty} \mu\left(h_{n}\right)=\mu\left(h_{0}\right)$ e $\left(f_{n}\right) C E$, uma sequência crescente de fun ções com $f_{n}$ convergindo para $f_{0}, f_{0} \in E$.

Tomando-se $h_{1}=f_{1}, h_{2}=f_{2}-f_{1}, \ldots h_{n}=f_{n}-f_{n-1}$ a sequência das reduzidas será: $s_{1}=f_{1}, s_{2}=f_{2}, \ldots s_{n}=f_{n}$. Como a sērie dada converge a sequência das reduzidas é convergente, então:

$\sum_{n=1}^{\infty} \mu\left(h_{n}\right)=\mu\left(f_{0}\right) ; \log 0 \mu$ converge para $\mu\left(f_{0}\right)$.
As proposições equivalentes do teorema anterior são as con dições de Lebesgue-Daniel1.

Definição

Seja $E C F(X ; \mathbb{R})$ um espaço vetorial e $\mu: E \rightarrow \mathbb{R}$ um funcional. $\mu \bar{e}$ uma integral inicial em $X$ se:

i) $E$ é um crã em $X$.

ii) $\mu . E \rightarrow \mathbb{R}$ ē um funcional linear positivo em $E$.

iii) $\mu$ satisfaz as condições de Lebesgue-Daniell.

Exemp Zo

Seja X um espaço localmente compacto de Hausdorff e $K(X ; \mathbb{R})$ o conjunto das funções contínuas de suporte compacto definidas em $X$. Todo funcional linear positivo $\mu: K(X ; \mathbb{R}) \rightarrow \mathbb{R} \bar{e}$ uma integral inicial.

Com efeito, tem-se que $K(X ; \mathbb{R})$ é um $c l \tilde{a}$ e $\mu$ um funcional linear positivo, logo basta mostrar que $\mu$ satisfaz uma das condições 
de Lebesgue-Daniell.

Seja $\left(f_{n}\right) C K(X ; \mathbb{R})$ uma sequência crescente de funções tal que sup $f_{n}=1 \lim _{n \rightarrow \infty} f_{n}=f_{0}, f_{0} \in K(X ; \mathbb{R})$. O suporte de $f_{n}, n=0,1,2 \ldots \bar{e}$ um compacto contido na união dos suportes de $f_{1}$ e $f_{0}$.

Seja $k=$ suporte $f_{1}$ união suporte $f_{0}$. $k$ é um compacto conti do em $x$ e suporte de $f_{n}$ está contido em $K$. Seja $x \in X$ tal que $f_{n}(x) \neq 0$. Se $f_{n}(x)<0$, então $f_{1}(x)<0$ logo $x$ pertence ao suporte discreto de $f_{1}$; se $f_{n}(x)>0$ então $f_{0}(x)>0$, logo $x$ pertence ao suporte discreto de $f_{0}$.

A sequência $\left(f_{n}\right)$ é crescente e $\left(f_{n / k}\right)$ isto é, $f_{n}$ restrita ao conjunto $K$ converge para $f_{0 / K}$, logo pelo teorema de Dini, a con vergência é uniforme em $K$. Como $k \bar{e}$ um compacto em $X$, existe um compacto $K^{\prime}$ contido em $X$ tal que $K$ estā contido no interior de $K^{\prime} e$ existe uma função $g: X \rightarrow \mathbb{R}$, contínua de suporte compacto $0 \leq \mathrm{g} \leq 1$, tal que :

$g(x)=1$ para todo $x \in K$ e
$g(x)=0$ para todo $x \in \overline{C K}$.

Então, dado $\epsilon>0$, existe $n_{0} \in \mathbb{N}$ tal que $\left|f_{n}(x)-f_{0}(x)\right|<\epsilon$ pa ra todo $x \in X$ e $n \geq n_{0}$, dai $\left|f_{n}-f_{0}\right| \leq \epsilon$. g. Como $\mu$ é um funcional $1 \underline{i}$ near positivo tem-se que $\left|\mu\left(f_{n}\right)-\mu\left(f_{0}\right)\right| \leq \mu\left(\left|f_{n}-f_{0}\right| \leq \epsilon_{\mu}(g)\right.$.

Exemp zo

Seja $X$ um espaço compacto de Hausdorff e $C(X ; \mathbb{R})$ o clã das funções reais continuas definidas em $X$. Todo funcional linear pos $\underline{j}$ tivo $\mu: C(X ; \mathbb{R}) \rightarrow \mathbb{R}$ é uma integral inicial em $X$.

Este exemplo é um caso particular importante do exemplo anterior.

Na terminologia de Bourbaki (3), $\mu$ é a medida de Radon po sitiva em $X$.

Teorema 3

$\operatorname{Seja} \operatorname{ECF}(X ; \mathbb{R})$ um clã e $\mu: E \rightarrow \mathbb{R}$ um funcional linear positi vo. São equivalentes as duas seguintes condições:

I) $\mu$ é uma integral inicial.

II) Se $\left(f_{n}\right) C E$ converge pontualmente para $f_{0} \in E$ e existe $g \in E$ tal que $\left|f_{n}\right| \leq$ p para todo $n=1,2, \ldots$ então $\mu\left(f_{n}\right)+\mu\left(f_{0}\right)$.

Demonstração

(I) implica (II). Sem perda de generalidade assume-se $f_{n}(x) \geq 0$ para todo $x \in X$ e para todo $n$ e $f(x)=0$ para todo $x \in X$.

Seja $g_{n, m}=m a-x\left(f_{n}, f_{n+1}, \ldots, f_{m}\right)$ para cada par de indices $m \geq n$. Então $0 \leq g_{n, m} \in E$ e $g_{n, m} \leq$ g para todo $m \geq n, g \in E$. 
Para cada $n$, a sequência $\left(g_{n, m}\right)$ é crescente e limitada em $m>n$. Para cada $\epsilon>0$ e para cada $n$, existe um $m_{n}>m$ tal que $m_{n}<m_{n+1}$ e $0 \leq \mu\left(g_{n, K}\right)-\mu\left(g_{n, m_{n}}\right) \leq \epsilon / 2^{n}$ para todo $k \geq m_{n}$.

Para simplificar põe-se $g_{n, m_{n}}=u_{n}$. Então $0 \leq \lim _{n \rightarrow \infty} \sup u_{n}(x)$ $\leq \lim _{n \rightarrow \infty} \sup f_{n}(x)=\lim _{n \rightarrow \infty} f_{n}(x)=0$ para todo $x \in X$. Para cada $n$, tem-se:

$0 \leq f_{n} \leq u_{n} \leq \min \left(u_{1}, \ldots u_{n}\right)+\sum_{i=1}^{n-1}\left(\operatorname{māx}\left(u_{i}, \ldots u_{n}\right)-u_{i}\right)$

Esta desigualdade è verdadeira porque para $1 \leq i \leq n$, $0 \leq u_{n}=u_{i}+\left(u_{n}-u_{i}\right) \leq u_{i}+\left(\operatorname{máx}\left(u_{i}, \ldots u_{n}\right)-u_{i}\right) \leq u_{i}+\sum_{k=1}^{n-1}\left(\operatorname{máx}\left(u_{k}\right.\right.$, $\left.\left.\cdots, u_{n}\right)-u_{K}\right)$.

Visto que $\operatorname{máx}\left(u_{i}, \ldots u_{n}\right)-u_{i}=\max x\left(f_{i}, \ldots f_{m_{n}}\right)-u_{i}=g_{i, m_{n}}-g_{i, m_{i}}$ e $m_{n}>m_{i}$ para $n>i$ conclui-se que, $\mu\left(m a \bar{x}\left(u_{i}, \ldots u_{n}\right)<\epsilon / 2^{i} \quad\right.$ para $1 \leq i \leq n$ e então para cada $n, 0 \leq \mu\left(f_{n}\right) \leq \mu\left(\min \left(u_{i}, \ldots u_{n}\right)+\epsilon \frac{1}{2^{n-1}}\right.$. Como $\lim _{n \rightarrow \infty} u_{n}(x)=0$ para todo $x \in X$, tem-se que a sequência $\left(\min \left(u_{1}, \ldots, u_{n}\right)\right)$ decresce para zero, logo para todo $x \in X, \lim _{n \rightarrow \infty} \mu$ (min $\left.\left(u_{1}, \ldots u_{n}\right)\right)=0$. Segue-se então que $\lim _{n \rightarrow \infty} \mu\left(f_{n}\right)=0$.

(II) implica (I). E imediato.

0 tipo de convergência considerada no item (II) do teore ma anterior é chanada de convergência dominada.

\section{A integral de Riemann como uma integral inicial}

Prova-se que a integral de Riemann sobre $[a, b]$ é uma inte gral inicial. Arzelà chegou a esse resultado, mas a denominaçãoē pos terior.

Preliminarmente, mostra-se que as integrais de Riemann das funções contínuas e das funções escalonadas sobre $[a, b]$ podem ser usadas para calcular a integral superior e a integral inferior de uma função limitada f sobre $[a, b]$.

\section{Esse è o conteūdo essencial do lema que segue.}

Lema

Para cada função limitada $f \geq 0$ e cada $\epsilon>0$ existe uma fun ção contīnua $g \in C([a, b] ; \mathbb{R})$ satisfazendo $0 \leq g \leq f$ e $\int_{a}^{b} f \leq s_{a}^{b} g+\epsilon$. Demonstração

Para cada $\epsilon>0$, existe uma função escalonada s em $[a, b]$ sa tisfazendo $0 \leq s \leq f e \int_{a}^{b} f \leq \int_{a}^{b} s+\epsilon / 2$.

A função escaT̄onada s pode ser transformada na função tra 
pezoidal g tal que, $0 \leq \mathrm{g} \leq \mathrm{s}$ e $\int_{a}^{b} \leq \int_{a}^{b}+\epsilon / 2$. segue-se então que existe uma função contīnua g com $0 \leq g \leq f e \int_{a}^{b} f \leq \int_{a}^{b} g+\epsilon$.

Teorema 4

Seja $\left(f_{n}\right)$ uma sequência decrescente de funções integrāveis em $[a, b]$. Se $\lim _{n \rightarrow \infty} f_{n}(x)=0$ para todo $x \in[\underline{a}, b]$ então $\lim _{n \rightarrow \infty} \int_{a}^{b} f_{n}(x) d x=0$, ou seja, a integral de Riemann $f \rightarrow \int_{a}^{b} f \bar{e}$ uma integral inicial sobre $R([a, b] ; \mathbb{R})$.

Demonstração

Segue do lema anterior que para cada $\epsilon>0$ e para cada $n$, existe uma função contĩnua $g_{n}$ tal que $0 \leq g_{n} \leq f_{n}$ e $\int_{a}^{b} f_{n}(x) d x \leq$ $\int_{a}^{b} g_{n}(x) d x+\epsilon / 2^{n}$.

Para cada $n$, seja $h_{n}=\min \left(g_{1}, \ldots g_{n}\right)$. Então $0 \leq h_{n} \leq f_{n}, h_{n} \quad \bar{e}$ contīnua, $n=1,2 \ldots$ e a sequência $\left(h_{n}\right)$ decresce para zero para todo $x \in[a, b]$. Pelo teorema de Dini, a sequência $\left(h_{n}\right)$ decresce para ze ro uniformemente em $[a, b], \log 0 \lim _{n \rightarrow \infty} \int_{a}^{b} h_{n}(x) d x=0$.

A prova do teorema estará concluida se for provada a des $\underline{i}$ gualdade:

$0 \leq \int_{a}^{b} f_{n}(x) d x \leq \int_{a}^{b} h_{n}(x) d x+E\left(1-\frac{1}{2^{n}}\right)$.

Como a sequēncia $\left(f_{n}\right)$ è decrescente tem-se que para cada $n, f_{n}=\min \left(f_{1}, \ldots, f_{n}\right)$ e então:

$0 \leq f_{n}-h_{n}=\min \left(f_{1}, \ldots, f_{n}\right)-\min \left(g_{1}, \ldots, g_{n}\right) \leq \sum_{i=1}^{n}\left(f_{i}-g_{i}\right)$.

Segue-se que para cada $n$,

$0 \leq \int_{a}^{b} f_{n}(x) d x-\int_{a}^{b} h_{n}(x) d x \leq \sum_{i=1}^{n} \int_{a}^{b}\left(f_{j}(x)-g_{i}(x)\right) d x \leq \sum_{i=1}^{n} \in / 2^{i}=$

$=E\left(1-\frac{1}{2^{n}}\right)$, o que conclui a demonstração.

Isso prova que a integral de Riemann $f \rightarrow f_{a}^{b} f$ sobre $R([a ; b]$; $\mathbb{R}$ ) satisfaz as condições de Lebesgue-Danie11, logo è uma integral inicial pois $R([a, b] ; \mathbb{R})$ è um cĩa.

CONCLUSAOO

Ficou comprovado neste trabalho que a restrição da inte gral de Riemann a todo $C l \tilde{a} E$ contido no $e l \tilde{a} R([a ; b] ; \mathbb{R})$ das funções reais definidas em [a,,$\left.b^{-}\right]$integrāveis à Riemann, é uma integral ini cial. Como os conjuntos $C([a, b] ; \mathbb{R}) ; D([a ; b] ; \mathbb{R}) ; \operatorname{Reg}([a, b] ; \mathbb{R}) e$ 
Esc $[a, b] ; \mathbb{R}$ ) são clãs de funções integrāveis, então a integral de Riemann elementar é uma integral inicial.

Num estudo posterior poderā obter-se a integral inicial as sociada a uma medida de Lebesgue, o prolongamento de Darboux de uma integral inicial e o teorema de Representação de Riesz, o qual afi $\underline{r}$ ma que, toda integral inicial $\bar{e}$ a restrição de uma Integral de Rié mann Abstrata, definida por uma medida inicial (2).

\section{BIBL IOGRAF IA}

1. ARZELA, C. SulZi Integrazione per Serie, Atti Acc. Lincei Rend, Rome, (4) 1(1885), 532-537.

2. BISOGNIN, E. A Integral de Riemann Abstrata e o Teorema de Repre sentação de Riesz. Rio de Janeiro. 1979. (Tese de Mestrado UFRJ).

3. BOURBAKI, N. Integration, Chap. I, II, III, IV. Paris. 1952.

4. LUXEMBURG, W.A.J. Arzelä's Dominated Convergence Theorem for the Riemann Integral. American Mathematical Monthly, 78(1971),970979.

5. RUDIN, W. Principles of Mathematical Analysis. Third Edition.Mc. Graw-Hi11.

Recebido em maio, 1981 ; aceito em novembro, 1981. 
